\title{
Multiple Multi-Objective Servo Design - Evolutionary Approach
}

\author{
Piotr Wozniak \\ Institute of Automatic Control, Technical University of Lodz \\ Poland
}

\section{Introduction}

Design of control systems is characterised by many targets, therefore the methods enabling optimisation of several objectives have received more and more attention over the past years. When dealing with multi-objective optimisation problems the notion of the scalar function optimality was extended. The most common approach was originally proposed in 19th century by Edgeworth and later generalised by Pareto. This trade-off approach means no element of the vector of optimal solution, so called Pareto optimal solution, can be improved without making some other criteria worse. There are many different notions of dominance. One of them is so called weak Pareto dominance relation which is defined as follows :

$$
\preceq_{\mathcal{F}^{\prime}}:=\left\{(\mathbf{x}, \mathbf{y}) \mid \mathbf{x}, \mathbf{y} \in X \wedge \forall f_{i} \in \mathcal{F}^{\prime}: f_{i}(\mathbf{x}) \leq f_{i}(\mathbf{y})\right\}
$$

where $\mathcal{F}^{\prime}$ is a set of objectives with

$$
\mathcal{F}^{\prime} \subseteq \mathcal{F}:=\left\{f_{1}, \ldots, f_{k}\right\}
$$

A solution $x^{*} \in X$ is called Pareto optimal if there is no other $x \in X$ that weakly dominates $x^{*}$ with respect to the set of all objectives taking into account all constraints. The set of all optimal solutions form the Pareto set.

Most of the research in the multi-objective optimisation has concentrated on tracing the Pareto front. Often this solution, which is non-dominated in the objective space, cannot be described analytically especially when the complexity of the problem makes exact methods unsuitable. The Pareto set is the projection of the Pareto front to the decision space.

In the last 20 years meta-heuristics approach to the multi-objective optimisation problems proved it can be applied even when only little is known about the underlying problems. From these methods, evolutionary algorithms are, without a doubt, the most widely used today mainly due to their flexibility while dealing with non-linear, non-quadratic, nonconvex problems and thanks to their ease of use (for extensive presentation of the state-ofthe-art research results see (Coello Coello, et al., 2007)). Also in engineering design formulated as multi-objective optimisation problems the evolutionary algorithms (MOEA) 
achieve popularity (Fleming et al., 2005) although generating Pareto front approximation is computationally expensive.

At the moment, thanks to rapid progress in computing technologies, novel algorithms of population-based optimisation may now be run on multiprocessor computing platforms in shorter time.

On the other hand, the designer, as well as the decision maker, may not be interested in having an excessively large number of Pareto optimal solutions (vectors from the decision space) to deal with due to overflow of information. Therefore, many multi-objective optimisation problems are reformulated to find a manageable number of Pareto optimal vectors which are evenly distributed along the Pareto front, and thus good representatives of the entire set of decisions. In real problems, a single solution must be selected.

Preferably, unique solution must belong to the non-dominated solutions set and must take into account the preferences of a designer and the decision maker.

Evolutionary methods are extensively applied for multi-objective optimisation problems mostly with two or three objectives only (Coello Coello, et al., 2007). On the other hand designers may prefer to put every performance index related to the problem as an objective, rather than as a constraint, thereby increasing number of criteria. The problems with a high number of objectives cause additional challenges with respect to low-dimensional problems. Current algorithms, developed for problems with a low number of objectives, have difficulties to find a good Pareto front approximation for higher dimensions. Even with the availability of sufficient computing resources, some methods are practically not useable for a high number of objectives. It has been investigated, whether it is possible to effectively solve optimisation problems with a large number of objectives where most of solutions generated become incomparable (Brockhoff \& Zitzler, 2006). In the complex design it is not clear whether any two given objectives are nonconflicting. That is, although a conflict exists elsewhere, some objectives may behave in a non-conflicting manner near the Pareto front. In such cases, the trade-off curve may be of dimension lower than the number of objectives.

The problem of dimensionality reduction multi-objective optimisation is defined as the question of finding a minimum objective subset, maintaining the given dominance structure (1) and good approximation of the Pareto front.

There are increasing number of research recently on influence of the objectives reduction on quality of the Pareto front approximation. In the literature dominates the a posteriori approach, where reduction is performed after preliminary solution to the multi-objective optimisation problems, (Deb \& Saxena, 2005), (Brockhoff \& Zitzler, 2006), (Woźniak, 2007a). Alternatively, a reduction in the complexity of most design problems is typically achieved by the problem decomposition based on the designer/decision maker's knowledge (Engau \& Wiecek, 2007), or the transformation of the multi-objective optimisation problem into the set of single-objective optimisation problems (Qingfu \& Hui, 2007).

The objective of this study is twofold. First, aim is to find a new coordination mechanism which guarantees that the final selection leads to a design that is Pareto optimal for the overall multiple Multi-Objective Optimisation Problem (mMOOP). The second aim is to propose a procedure for the mMOOP with many objectives solution under the changing environment conditions.

The methodology presented in this study integrates several multi-objective optimisation problems, while steering clear of the high dimensionality problems. 
The issues of multi-objective optimisation are highlighted in Section 2. The multiple multiobjective optimisation problem is outlined in Section 3 while the proposed algorithm for the mMOOP solution is proposed in Section4. In Section 5 the application of the mMOOP design is presented for the servo design as a future field of interest. The Section 6 summarizes the study.

\section{Dimensionality issues in multi-objective optimisation}

The majority of the existing multi-objective evolutionary algorithms for approximating the Pareto front have been designed for, and tested on, low dimensional examples (Coello Coello, et al. 2007). However, for complex optimisation problems often a higher number of dimensions occur. Increased number of criteria cause difficulties in terms of the quality of the Pareto front approximation and running time (e.g. algorithms based on the hypervolume indicator (Brockhoff \& Zitzler, 2006) lead to running times exponential in the number of objectives). Additionally there is a greater probability of having any two arbitrary solutions to be non-dominated to each other. Consequently the proportion of such solutions in the population increases. Since multi-objective evolutionary algorithms put more emphasis on the non-dominated solutions, a significant part of the old population is preserved in the elite (Coello Coello, 2007). Therefore growing elite leaves no much room for new solutions to be included in the population when the constant size of pool is assumed. This, in consequence, reduces the selection pressure for the better solutions in the population and the search process slows down.

When the Pareto dominance-based ranking procedures become ineffective determining the quality of solutions, new measures and relations are introduced to guide the optimisation process. Recent results on using preference order-based approach as an optimality criterion in the ranking stage of multi-objective evolutionary algorithms (Engau \& Wiecek, 2007) proved convergence improvement.

In general dimension reduction aims at keeping those objectives that can explain most of the variance in the objective space. However, it is not clear :

i. how the objective reduction alters the dominance structure,

ii. what is the quality of a generated objective subset.

The most accepted method is aggregation of the vector objectives into the single criterion by introducing the weighted sums. The multi-objective problem is therefore reduced to single function optimisation which is easy to solve even in the presence of local optima and, on a first sight, scale well.

But for high dimensions these techniques reach their limits, since :

i. it is hard (or even impossible) to determine good weights,

ii. such approaches lack the desired parallel search ability.

Another prospective ways of solving this type of problems includes reduction in the number of objectives (Brockhoff \& Zitzler, 2006), (Woźniak \& Witczak, 2007), (Woźniak, 2007a) or discovering objectives, which are entirely unrelated by the divide-and-conquer strategy (Purshouse \& Fleming, 2003). The later method is based on splitting multi-objective optimisation problem into sub-problems. The main limitation of this approach is excessive number of pair-wise comparisons at the merge step after solution of sub-problems.

Decomposition methods are particularly well suited for design optimisation as most of complex engineering systems usually consist of many subsystems and components having smaller complexity. Dividing large and complex systems into several smaller entities is done 
to enable local optimisation and decision-making. In general, however, these subsystems will still be coupled so that the solution of each subsystem is dependent upon information from the others. Hence, along with the benefit of reduced complexity, comes the issue of exchange of the separate design decisions (i.e. values of the criteria arguments) to eventually arrive at a single overall design solution that is feasible. To solve this coordination problems the concept of the multiple multi-objective optimisation is introduced in Section 3.

\section{Problem definition}

The mathematical background of the multiple multi-objective optimisation problem remains the same as of a classic multi-objective optimisation problem.

We consider the common formulation of the multi-objective optimisation problem in its general form :

$$
\begin{aligned}
& \text { min. } f(x), \\
& \text { s.t. } \quad x=\left[x_{1}, x_{2}, \ldots, x_{m}\right], \\
& x \in X \subseteq S \subseteq R^{m} \\
& f(x)=\left[f_{1}(x), f_{2}(x), \ldots, f_{n}(x)\right] ; n \geq 2 \\
& \text { subject to. } g(x)=\left[g_{1}(x), g_{2}(x), \ldots, g_{m}(x)\right] \leq 0, \\
& h(x)=\left[h_{1}(x), h_{2}(x), \ldots, h_{l}(x)\right]=0,
\end{aligned}
$$

where $x$ is the vector of the decision variable, which might be subject to inequality $g(x)$ and/or equality constraints $h(x)$.

A solution which satisfies all the constraints is called a feasible one. Due to contradicting objectives there is no single solution to (2). Instead there is a set of alternative solutions.

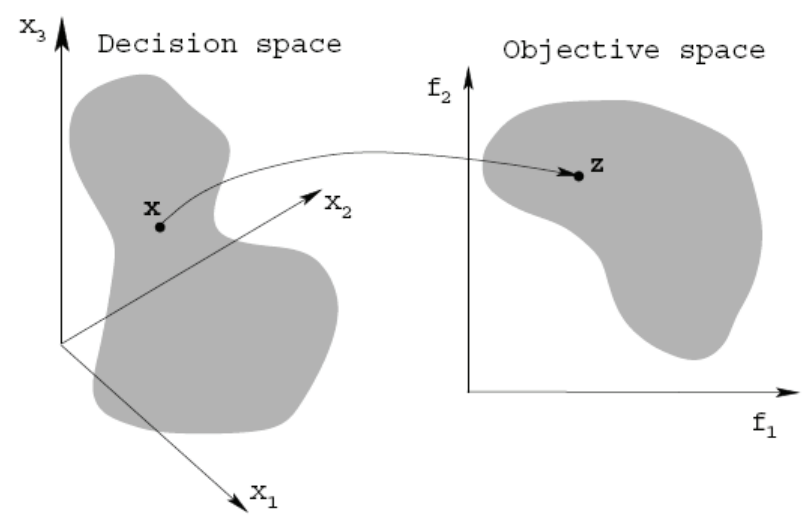

Fig. 1. Representation of the decision space and the corresponding objective space.

These solutions are optimal in the sense that no other solutions dominate (are superior to) them when all objectives are considered. They are known as Pareto-optimal solutions.

The interest, in the classical multi-objective optimisation problem, is therefore on the tradeoffs with respect to the objectives (Shukla \& Deb, 2007). Each objective function maps 
the input decision vector (point in the $\mathrm{m}$ dimensional decision space) (see Fig. 1) to the target vector in the $\mathrm{n}$ dimensional objective space.

The domination relation defined in the objective space is used to identify

i. the Pareto set in the decision space,

ii. the Pareto front in objective space and

iii. the Pareto rank of each solution.

The main difference between approach introduced in this study and classical single multiobjective optimisation problem lies in the synchronised consideration of simultaneous multi-objective optimisation problems sharing the same decision space, but with the environment changes. Distinct environment conditions may be introduced when variations in the multi-objective optimisation problem formulation is needed to describe discrepancy between the physical plant and the mathematical model with constraints used for the design.

Every vector of the environment changes form the context which therefore is identified by its parameters, and is denoted $c$. The context belongs to the permissible environment conditions space $\mathrm{C}^{0}$.

There are several possible ways to integrate environment conditions $c \in C^{0}$ into a classical multi-objective optimisation problem. In each case the vector of objective functions (results in Fig.2) changes.

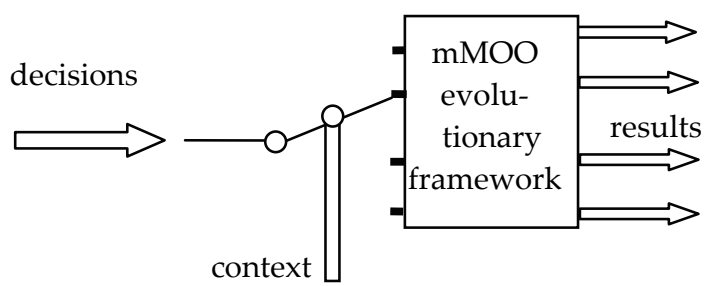

Fig. 2. The changes of environment conditions for the plant leading to multiple multiobjective optimisation problem (mMOOP).

The alternatives may be obtained by :

i. extending the decision (input) vector by the context $c$. Now we consider the resulting mapping with extended (comparing to (2)) arguments $f^{*}(\mathrm{x}, \mathrm{c})$. A common algorithm for a multi-objective optimisation problem is used to find all optimal solutions in the decision space of the higher dimension. Since the decision space of the problem and the context space $C^{o}$ are unified, just the optimal solutions $x_{c}^{*}$ over the new input space will be found. For this reason such integration of the environment conditions is not suitable for the control system design.

ii. extending the objective vector by the context $\mathrm{c}$. The resulting mapping will be $f_{c}(\mathrm{x})$ with $f_{c} \in F C^{n+o}$ in higher dimensional space. A common algorithm for a single multi-objective optimisation problem is used to find all optimal solutions in the objective space of the higher dimension. For this reason, as discussed in details in Section 3, such an integration of the context is not preferred. 
iii. treating every context as a single multi-objective optimisation problem. This corresponds to an exhaustive a-posteriori search in every o approximated Pareto fronts (for all possible contexts). It is obvious that such an approach is not efficient, because it leads to optimisation in the set of o fronts $f_{c}\left(\mathrm{x}_{\mathrm{c}}\right)$.

iv. The multiple multi-objective optimisation problem mapping. The characteristic is that all different multi-objective optimisation problems share the input space, and the outputs are generated concurrently $f_{c}(\mathrm{x})$.

The key observation is that in the multi-objective optimisation problem framework iv. finding Pareto optimal solutions is equivalent to a search for a trade-off solution with variation within some parameters.

In this study variations included in the multiple multi-objective optimisation problem mapping formulation iv. are considered as distinct working conditions of the system (see Fig.2).

Directly from the above definitions of the multiple multi-objective optimisation problem mapping follows that there are multiple outputs for a single decision input (one for every context). After collecting a set of solutions, the Pareto rank for every solution in each context can be calculated.

To compress this information to a single value only the highest Pareto rank value (the lowest from the calculated $\operatorname{Prank}_{c^{i}}$ ) is selected and further defined as

$$
\text { bPrank }=\min \left\{\operatorname{Prank}_{c^{l}}, \operatorname{Prank}_{c^{2}}, \ldots, \operatorname{Prank}_{c^{o}}\right\}
$$

This value bundles the quality of a solution into a single value. As a result its value is crucial for multi-objective optimisation algorithms, because they are based on ranking comparisons of different solutions.

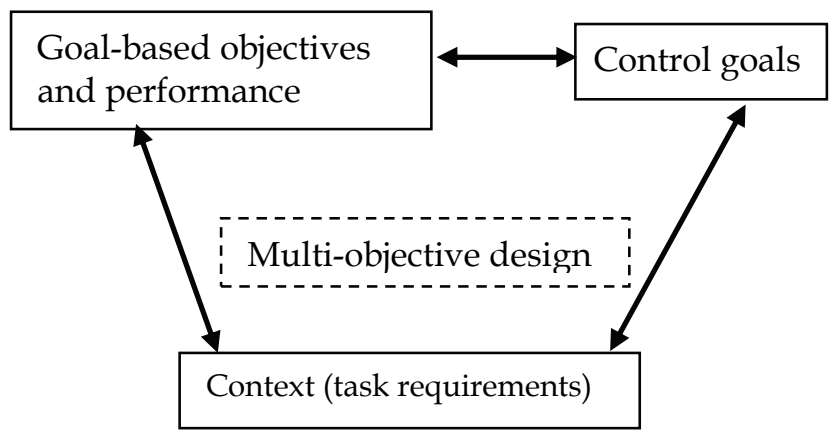

Fig. 3 Multi-objective control design framework with task requirements - context.

In this work, we propose a procedure of transferring some performance criteria of the control system into the context variables. The approach is motivated by the real-life problem of having a large number of potential objectives in the redundant robot manipulators control based upon the existing multi-criteria inverse kinematics, and will be discussed in details in Section 5.

The task-based controller is a controller that unifies position and force control of redundant manipulators and takes task requirements as the central component of the multi-objective control design framework, with context presented in Fig. 3. 


\section{Evolutionary methodology of the multiple multi-objective optimisation problem solution}

Since evolutionary algorithms deal with a number of population members in each generation, they are ideal for finding multiple Pareto-optimal solutions in of the multiobjective optimisation problem. All of these methods emphasize :

i. non-dominated solutions for progressing towards the Pareto-optimal front,

ii. less-crowded solutions for maintaining a good diversity among obtained solutions,

iii. elites to provide a faster and reliable convergence near the Pareto-optimal front.

There are numerous approaches for solving multi-objective optimisation problems. The salient features of multi-objective evolutionary algorithms are :

i. the convergence of solutions in the objective space to the Pareto front,

ii. support for diversity of the solutions along the front,

iii. efficiency characterised by the processing time or the number of evaluations required.

New algorithms introduced every year aim to improve on one or more of the above mentioned issue. Some of the most well-known algorithms are: VEGA, MOGA, PAES, NSGA-II and SPEA2. For comprehensive description see (Konak et al., 2006) and (Coello Coello et al., 2007).

Essential parameters to be fixed in an evolutionary algorithm:

i. population size,

ii. number of generations,

iii. parameters related to selection,

iv. recombination (crossover probability, crossover operator),

v. mutation (mutation probability, mutation operator).

Population size is a crucial parameter in a successful application of each algorithm. Even in the case of an adequate population size optimisation the algorithm must be run for a critical number of generations in order to obtain convergence near the optimal solution (Coello Coello et al., 2007).

In case where context can be configured concurrently, a single evaluation run delivers several results, each consisting of multiple objective values, for each instance of the multiobjective optimisation problem.

The presented approach is based on sequential calculations of MOO sub-problems of the multiple multi-objective optimisation problem. After selecting one, leading multiobjective optimisation problem, its Pareto set is henceforth considered as constant for all remaining multi-objective optimisation problems.

The idea behind this approach is presented in Fig. 4 for two contexts of a bi-objective problem (denoted $f_{1}{ }^{1} f_{2}{ }^{1}$ in Fig. 4 a and $f_{1}^{2} f_{2}{ }^{2}$ in Figs. $4 \mathrm{~b}$ and $4 \mathrm{c}$, respectively).

After four elements of the Pareto front for the first context are found and designated with different symbols in Fig. 4a, their arguments in the decision space are passed to the second context. Using each of the values may result in a front shown in Fig. $4 \mathrm{~b}$, when the next, second, multi-objective optimisation problem is solved. This means that for each point in the objective space of the first multi-objective optimisation problem there may be more than one solution in the second objective space. These are designated by the same symbols like in Fig. 4a.

In the next step the results are sorted for non-dominancy and lead to the front depicted in Fig. 4c (dominated solutions are discarded). 


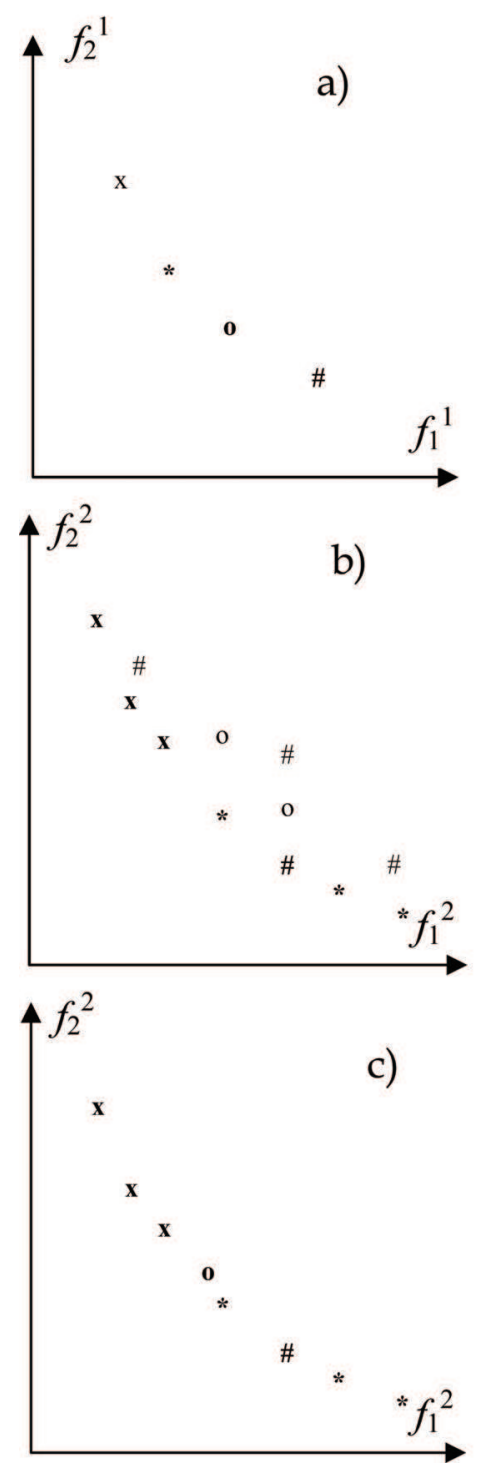

Fig. 4 Outline of Pareto front derivation for two contexts of bi-objective optimisation problems

Considering the above mentioned approach, the pseudo-code of the proposed sequential optimisation may be formulated as presented in Fig. 5 .

For this specific multiple multi-objective optimisation problem design the order of the considered sequences of contexts is far less important than in the similar multiple multiobjective optimisation problem s proposed in (Avigad, 2007) and (Ponweiser \& Vincze, 2007). It is possible to make it robust to the order of the multi-objective optimisation 
problems by introducing epsilon tolerances to reflect the implicit trade-off between solutions of two different contexts.

1. Decision Making step - identify all contexts $C_{i}, i=1, \ldots, 0$, and introduce the order in the $C$ set.

2. Initialise parameters of MOEA and search space.

3. Apply MOEA with non-dominated sorting to solve $C_{1}$. Store results in form of the Pareto set $x_{1}$ and the Pareto front $c_{1}$, i.e. $\left(x_{1}, t_{1}\right)$.

4. For $j:=i+1$ to $o$ do

a. Initialise $C_{j}^{\text {th }}$ MOEA parameters taking into account Pareto solutions $\left(x_{j-1}, c_{j-1}\right)$

b. Apply MOEA with non-dominated sorting to solve $c_{j}$. Store results in form of the Pareto set $x_{j}$ and the Pareto front $c_{j}$, i.e. $\left(x_{j}, c_{j}\right)$

c. Reject from $\left(x_{j-1}, c_{j-1}\right)$ solutions, which became dominated in the $j^{\text {th }}$ step

5. IF the maximal number of populations is reached THEN STOP ELSE goto STEP 3

Fig. 5 Pseudo-code of the proposed mMOOP algorithm.

Solving the individual MOO sub-problems before selecting a final design generally may overemphasize one context, while significantly degrading the performances of others. Moreover, it is shown that the best compromise solution is not necessarily optimal for any MOO sub-problem, and thus remains unknown to the designer who follows the traditional decomposition - integration approach. We plan to consider this issue in the near future.

The first and probably the most important property that needs to be considered for the design of optimiser for a multiple multi-objective optimisation problem are multiple instances of the objective space. There exists one for every context. Although any of averaging technique can be used to operate in these spaces (e.g. mean, standard deviation, minimum or maximum value), a careful selection of values from each one is needed. Furthermore, the computational effort increases enormously because the calculations have to be done for every context separately. Out of these insights it is advisable to avoid performing any operations in the objective space.

In classical multi-objective evolutionary algorithms methods the objective space is intuitively used to calculate the density of solutions (for example in SPEA2 or NSGA-II). A solution for the multiple multi-objective optimisation problem is to relocate the density calculations from the objective space to the decision space. The placement of these measures, either in the decision space or in the objective space, was subject to a long scientific discussion (Coello Coello et al., 2007). In most of the implementations the objective space is used. Therefore, at this stage of research on multiple multi-objective optimisation problem, the NSGA-II (Deb, 2001) state-of-art algorithm is considered as the most prospective. 
Another effect that needs to be considered is the extension of the Pareto rank to the best Pareto rank (3). In the NSGA-II the Pareto rank is the main selection criteria. A drawback of the best Pareto rank is its computational effort, but so far no better approach may be put forward. The complexity of a single Pareto rank calculation is multiplied by the number of contexts. This issue still lacks a computationally effective solution.

\section{Multiple multi-objective optimisation problem of servo control - an outline}

We will consider the so-called mechatronic servo system, i.e. the servo system adopted in the numerical control machine or industrial robot with many joints. Generally, dynamic characteristics of robot actuators and sensors are highly nonlinear with constraints, and these factors cause trajectory control errors. Feeding back the difference between the robot servomechanism velocities enables force adjustment.

The performance criteria for robot control optimisation may be broadly divided into two categories :

i. constraint-based criteria,

ii. operational goal-based criteria.

The constraint-based criteria, as its name implies, are directly associated with system constraints (e.g. joint limits, obstacles, singularities, etc.). Therefore, in general they have clear physical meanings that the user can easily relate to. They are task-dependent and usually give more insight to the operator on the task at hand.

Operational goal-based criteria, on the other hand, are concerned with the ability of the robot to perform the task better. They are functions of only manipulator configuration and states, and are not tied to any specific task. This makes the criteria very useful for the system designer, who cannot foresee all the possible tasks the robot could perform in the future.

The comprehensive description of the objectives, and performance criteria, for optimisation of redundant robot system presented hereafter was published in the Ph.D. thesis (Pholsiri, 2004). Redundancy, in this context, is defined as having more inputs than those required to create the desired output. As such, traditionally non-redundant robots, e.g. most 6 degrees of freedom (DOF) commercial robots, can be considered redundant too if their tasks at hand require fewer DOFs than the robots possess. Redundancy implies an ability to change configuration of the joint without changing the position of the robot's end-effector.

The main criteria are listed hereafter, and will enable the introduction and formulation of the multiple multi-objective optimisation problem :

C1 Criteria for Joint Range Availability (JRA).

Every joint in a manipulator has its travel limits which cannot be exceeded. Any attempt to move a joint over its limit can potentially damage the robot.

$$
\gamma_{J R A}=\frac{1}{n} \sum_{i=1}^{n}\left(\frac{\left|\theta_{i}-\theta_{i, \text { mid }}\right|}{\theta_{i, \max }}\right)^{p}
$$

where :

$\theta_{i}$ is the joint displacement,

$\theta_{i, \text { mid }}$ is the displacement at the midpoint of the travel range,

$\theta_{i, \max }$ is the displacement at the travel limits. 


\section{C2 Criteria for Velocity Limit Avoidance.}

The joint Velocity Limit Avoidance (VLA) tries to minimise the velocity of each joint or the sum of the velocities of all joints. The velocity limit can be avoided by minimizing the norm of the joint velocity vector. It is crucial to keep VLA from approaching 0 . The pseudo inverse solution minimises the VLA criterion.

\section{C3 Criteria for Peak Torque Avoidance.}

Although their formulation is simple and straightforward, their use in practice is limited for various reasons. First of all, the torque readings require that torque sensors be present at all actuators, which is not common (due to their cost). Secondly, even with the torque information available, this criterion can only be used to monitor the torque states of the robot but generally cannot be used in redundancy resolution to prevent the robot from exceeding their joint torque limits because most, if not all, redundancy resolution techniques do not work in the force domain.

\section{C4 Criteria for Obstacle Avoidance.}

When a manipulator is utilised in a cluttered environment or in a multi-arm system, the need to avoid obstacles or contacts with other manipulators arises. This may be formulated in the form that it is independent of the number of links and the number of obstacles.

\section{C5 Criteria for Mathematical Singularity Avoidance.}

Physically, at singularities, a manipulator loses one or more degrees of freedom. The robot may not be able to move along the desired direction. To avoid mathematical software failure, it is crucial to keep MSA from approaching zero.

The objectives mentioned above (C1 - C5) represent constraint-based criteria and may compose the context for operational goal-based objectives (Gi).

The most important goal-based objectives are :

\section{G1 Criteria for Manipulator Precision.}

A manipulator's joints are expected to have some amount of error, including position sensor error (encoder resolution or noise), control error, and deflection due to joint compliance. These joint errors are propagated through the links and to the end effector. Minimizing the effect of this error propagation is essential in applications requiring precise manipulation.

\section{G2 Criteria for Speed of Operation.}

Maximising Velocity Transmission Ratio (VTR) will minimise the joint velocity required to produce a given end effector speed in the direction, in general or for any given joint velocity.

\section{G3 Criteria for Load Carrying Capacity.}

Maximizing Force Transmission Ratio (FTR) will increase the end effector force capability in the desired direction. Looking at formulations of the VTR and the FTR, it can be concluded that they are not independent.

\section{G4 Criteria for Energy Minimisation.}

Kinetic energy minimisation is one of the early criteria used in redundancy resolution because kinetic energy is directly associated with the power consumed by the system during its operation. It is desirable to minimise the energy-based objective, especially for repetitive tasks. 
A quick look at the list of performance criteria (G1 - G4) reveals that most, if not all, of these criteria are coupled. It is therefore not possible to optimise one criterion without affecting another. Hereafter there is a list of the major interaction between criteria. For example, maximising the JRA (4) criterion will likely have an impact on the VTR criterion. Even though the intention of adding the JRA to the redundancy resolution process is merely to avoid the joint limits, we may unintentionally decrease the ability of the robot to move in a desired direction. These couplings also make it impossible to completely separate the purposes of these criteria, making the task of choosing criteria for a given optimisation very difficult.

These couplings result in conflicts among criteria. The best example is the conflict between the speed and force capabilities of the robot. When considering them independently one would like to maximise both of them. However, because of the conflicting nature of these two quantities, it is physically impossible to do so at the same time. A closer look at the VTR and the FTR criteria shows that these two criteria are tightly coupled. As a matter of fact in some special cases they are the reciprocals of each other. It was investigated whether the VTR can be used to either increase the end effector speed or the end effector precision (Pholsiri, 2004). However, while increasing the speed requires that VTR be increased, improving the end effector precision demands the opposite.

These conflicts also cause difficulty when choosing appropriate criteria for a given task. The problems of couplings and conflicts among performance criteria are one of the main motivations behind the multi-objective optimisation research in the robot's servo control design.

In the considered redundant robot control problem the context is defined by constraintbased criteria (C1-C5).

While it is essential to keep the system from violating constraints (C1-C5) during operation, their values are not objectives of optimisation. Instead, their values may differ from one context to another. The most straightforward approximation is to keep every constraint constant during optimisation in each context.

At the present moment the investigation on the proposed novel multiple multi-objective optimisation problem is at its early stage of development. First simulation experiments showed that there is still significant potential for improvement, especially in the development of metrics measuring the performance of optimisation algorithms for multiple multi-objective optimisation problem in decision space, instead of using evaluation in the objective spaces (one space per context).

\section{Case study - servo design}

The mechatronic servo system, i.e. the servo system adopted in the numerical control machine or industrial robot is considered. In this system, there are two types of control. One is position control (PTP: point to point) emphasizing the arriving time and stop position from any position without considering the response route. Another is the contour control emphasizing the motion trajectory from the current position to the next position (position at each moment and its motion velocity).

The typical system includes the servo system of each axis, which is consists of the following parts : 
- the motor,

- the power amplifier,

- the current control,

- the velocity control,

- the position control.

The structure of the system is generally different from the servo system introduced in textbooks of automatic control and is presented in Fig. 5 (Woźniak, 2007b).

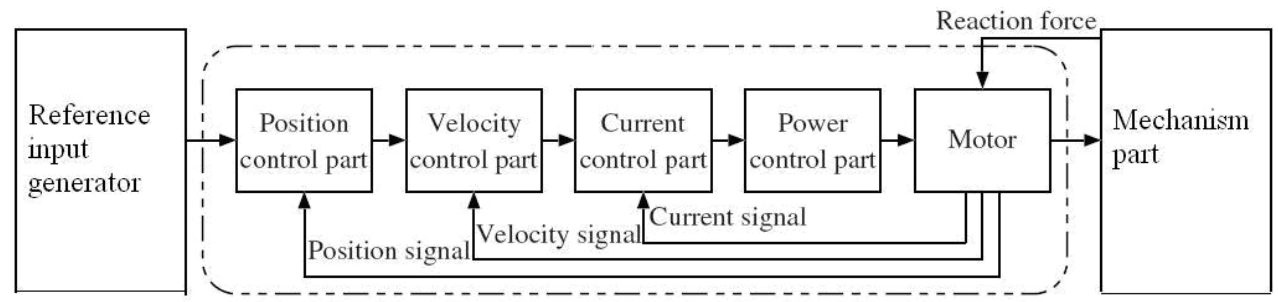

Fig. 5. Mechatronic servo system structure

\subsection{The comprehensive presentation of three multi-objective problems}

The overall design problem may be considered mMOOP with divided into three MOOPs as outlined in Fig.6.

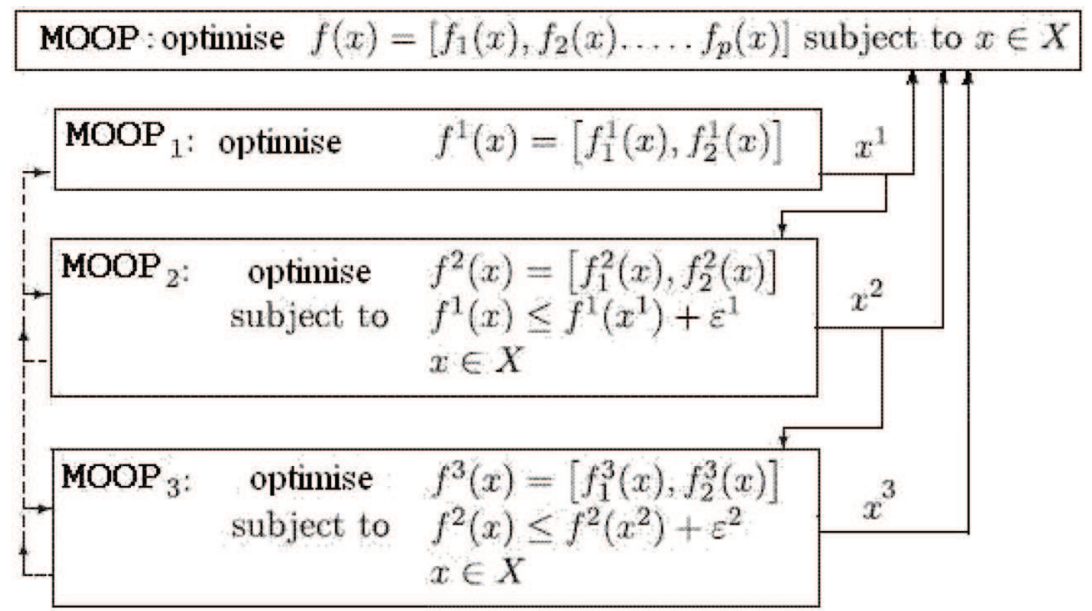

Fig. 6 The epsilon tolerance integration of the mMOOP with distinct contexts

The control goals may be easily organised in the same manner as presented in Fig.5. It is realistic, from engineering point of view, to consider position control part of the design as the most important one. This loop is responsible for the following the reference path with at last two conflicting targets - fast transients and small overshoot combined with the zeroing steady-state error. The position control loop supervises velocity signal control. The dynamics of this subsystem also has at least two conflicting objectives. 
The most inner part of the presented in Fig. 5 servo system structure has the most complex dynamics forced by the pulse-width modulation control of the permanent magnet synchronous motor. Unlike the mechanical control loops(i.e. Velocity signal, and Position signal), this one has to be modeled by discrete-time model with time constants of several microseconds.

The mMOOP interaction between multi-objective designs takes into account some tolerance $\varepsilon$, which improves robustness of the solution (Engau \& Wiecek, 2007) and is realised according to the coordination scheme outlined in Section 4 (see Fig.4).

\section{Conclusions}

This study contributed a novel formulation to the emerging research area of the optimisation methods - the multiple multi-objective optimisation problem. It is an extension of the multi-objective optimisation ideas to the set of concurrent multi-objective optimisation problems defined by changing the environment conditions - the context.

In this study, the burden of high dimensional multi-objective optimisation problem (as discussed in Sect. 3) is relaxed by considering aggregation of the constraint-based criteria with conditions for operational goal-based objectives.

The Pareto optimal solutions of the multiple multi-objective optimisation problem are evaluated without introducing ordering of the multi-objective optimisation problems. The shared decision space of multi-objective optimisation problems is considered as a connecting bridge between all multi-objective optimisation problems.

As an example from the control servo system design, the redundant robot design problem is outlined for further research.

In the future work, we intend to further investigate the information that can be obtained from the proposed trade-off and sensitivity analysis. In view of the current approach, we are aware of the remaining weakness that this information only allows a local trade-off assessment, and thus cannot be used for more accurate estimates in a larger region of the outcome space.

We would also like to address remaining issues such as computational benchmarking or further analysis of effects from grouping and ordering of objectives using examples from the industry. We believe that such future efforts will further improve the recognised features of the current method and eventually provide an effective and flexible decisionmaking tool for multi-objective design optimisation.

\section{References}

Avigad, G. (2007). multi-Multi-Objective Optimization Problem and Its Solution by a MOEA, Proceedings of the 4th Conference on Evolutionary Multi-Criterion Optimization - EMO 2007, Obayashi S. et al. (Eds.), pp.847-861, ISBN 978-3-54070927-5, Matsushima, Japan, March 5-8, 2007, Springer, Berlin

Brockhoff, D. \& Zitzler E. (2006). Are All Objectives Necessary? On Dimensionality Reduction in Evolutionary Multiobjective Optimization, Proceedings of the 9th Conference on Parallel Problem Solving from Nature-PPSN IX, Runarsson, T.P. et al. 
(Eds.), pp. 533-542, ISBN 978-3-540-38990-3, Reykjavik, Iceland, September 9-13 2006

Coello Coello, C.A. ; Lamont, G.B. \& Van Valdhuizen, D.A. (2007). Evolutionary Algorithms for Solving Multi-Objective Problems (2nd Ed.), ISBN 978-0-387-332549-3, Springer, Berlin

Deb, K. (2001) Multi-Objective Optimization Using Evolutionary Algorithms, Wiley, ISBN 978-0471-87339-6, Chicester, UK

Deb, K. \& Saxena, D.K. (2005) On finding pareto-optimal solutions through dimensionality reduction for certain large-dimensional multi-objective optimization problems. Technical Report KanGAL Report No. 2005011, Kanpur Genetic Algorithms Laboratory, 2005

Engau, A. \& Wiecek, M.M. (2007). 2D decision-making for multicriteria design optimization, Structural and Multidisciplinary Optimization, Vol.34, No.4, (Oct. 2007), pp. 301-315, ISSN 1615-147X

Fleming, P. J.; Purshouse, R. C. \& Lygoe, R. J. (2005). Many-Objective Optimization:An Engineering Design Perspective, Proceedings of the 3rd. Conference on Evolutionary Multi-Criterion Optimization - EMO 2005, Coello Coello, C. A. et al. (Eds.), pp. 14-32, ISSN 0302-9743 Guanajuato, Mexico, March 2005

Konak, A.; Coit, D.W. \& Smith, A.E. (2006). Multi-objective optimization using genetic algorithms: A tutorial, Reliability Engineering and System Safety, Vol.91, pp.992-1007, Sept.2006

Pholsiri, C. (2004). Task-based decision making and control of robotic manipulators Ph.D. Thesis, University of Texas at Austin, 2004, Available at: https://www.lib.utexas.edu/etd/d/2004/pholsirid74234/pholsirid74234.pdf

Ponweiser, W. \& Vincze, M. (2007) “The Multiple Multi Objective Problem - Definition, Solution and Evaluation, Proceedings of the 4th Conference on Evolutionary MultiCriterion Optimization - EMO 2007, Obayashi, S. et al. (Eds.), pp. 877-892, ISBN 9783-540-70927-5, Matsushima, Japan, March 5-8, 2007, Springer, Berlin

Purshouse, R. C. \& Fleming, P. J. (2003) An adaptive divide-and-conquer methodology for evolutionary multi-criterion optimisation, Proceedings of the 2nd Conference on Evolutionary Multi-Criterion Optimization EMO 2003, Fonseca, C.M. et al. (Eds.), pp. 133-147, ISBN 978-3-540-01869-8, Faro, Portugal, April 8-11, 2003, Springer, Berlin

Qingfu, Z. \& Hui, L. (2007) MOEA/D: A Multiobjective Evolutionary Algorithm Based on Decomposition, IEEE Trans on Evolutionary Computation, Vol. 11, No. 6, (Dec. 2007), pp.712-731, ISSN 1089-778X

Shukla, P.K. \& Deb, K. (2007) On finding multiple Pareto-optimal solutions using classical and evolutionary generating methods, European Journal of Operational Research, Vol.181, No. 3, (Sept 2007), pp.1630-1652, ISSN 0377-2217

Woźniak, P. (2007a) Dimensionality Reduction in Evolutionary Multiobjective Design: Case Study, Proceedings of the 9th annual conference on Genetic and evolutionary computation GECCO-2007, Thierens et al. (Eds.), pp. 913-915, ISBN 978-1-59593-697-4

Woźniak, P. (2007b) Multiple Multi-Objective Optimisation of Servomechanism Control Systems Design - Evolutionary Approach, Proceedings of the 13th IEEE International 
Conference on Methods and Models in Automation and Robotics MMAR 2007, Emirsajłow, Z. (Ed.), pp. 379-385, Szczecin, Poland, August 27-30, 2007

Woźniak, P. \& Witczak, P. (2007). Dimensionality Reduction in Evolutionary Multiobjective Design of Permanent Magnet Generator, Proceedings of the 4th Conference on Evolutionary Multi-Criterion Optimization EMO 2007, Obayashi S. et al. (Ed.), pp. 63-68, LBP, Matsushima, Japan, March 5-8, 2007 


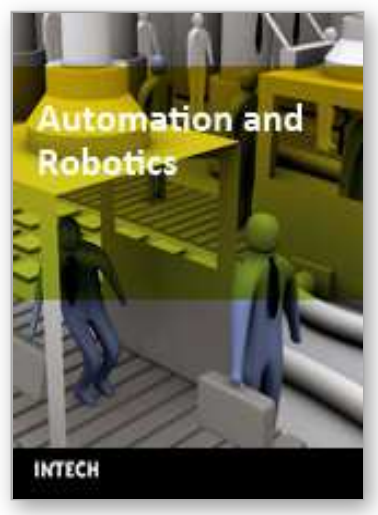

\author{
Automation and Robotics \\ Edited by Juan Manuel Ramos Arreguin
}

ISBN 978-3-902613-41-7

Hard cover, 388 pages

Publisher I-Tech Education and Publishing

Published online 01, May, 2008

Published in print edition May, 2008

In this book, a set of relevant, updated and selected papers in the field of automation and robotics are presented. These papers describe projects where topics of artificial intelligence, modeling and simulation process, target tracking algorithms, kinematic constraints of the closed loops, non-linear control, are used in advanced and recent research.

\title{
How to reference
}

In order to correctly reference this scholarly work, feel free to copy and paste the following:

Piotr Wozniak (2008). Multiple Multi-Objective Servo Design - Evolutionary Approach, Automation and Robotics, Juan Manuel Ramos Arreguin (Ed.), ISBN: 978-3-902613-41-7, InTech, Available from: http://www.intechopen.com/books/automation_and_robotics/multiple_multi-objective_servo_design__evolutionary_approach_

\section{INTECH}

open science | open minds

\author{
InTech Europe \\ University Campus STeP Ri \\ Slavka Krautzeka 83/A \\ 51000 Rijeka, Croatia \\ Phone: +385 (51) 770447 \\ Fax: +385 (51) 686166 \\ www.intechopen.com
}

\author{
InTech China \\ Unit 405, Office Block, Hotel Equatorial Shanghai \\ No.65, Yan An Road (West), Shanghai, 200040, China \\ 中国上海市延安西路65号上海国际贵都大饭店办公楼 405 单元 \\ Phone: +86-21-62489820 \\ Fax: +86-21-62489821
}


(C) 2008 The Author(s). Licensee IntechOpen. This chapter is distributed under the terms of the Creative Commons Attribution-NonCommercialShareAlike-3.0 License, which permits use, distribution and reproduction for non-commercial purposes, provided the original is properly cited and derivative works building on this content are distributed under the same license. 